\title{
Giant unilamellar vesicles - a perfect tool to visualize phase separation and lipid rafts in model systems
}

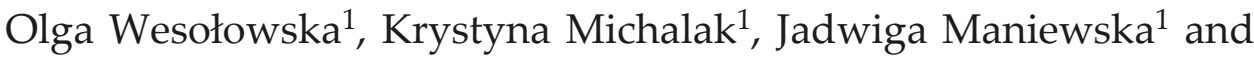 \\ Andrzej B. Hendrich ${ }^{2 \bowtie}$ \\ ${ }^{1}$ Department of Biophysics, and ${ }^{2}$ Department of Biology and Medical Parasitology, Wrocław Medical University, \\ Wroctaw, Poland
}

Received: 19 January, 2009; revised: 10 March, 2009; accepted: 13 March, 2009

available on-line: 17 March, 2009

\begin{abstract}
Model systems such as black lipid membranes or conventional uni- or multilamellar liposomes are commonly used to study membrane properties and structure. However, the construction and dimensions of these models excluded their direct optical microscopic observation. Since the introduction of the simple method of liposome electroformation in alternating electric field giant unilamellar vesicles (GUVs) have become an important model imitating biological membranes. Due to the average diameter of GUVs reaching up to $100 \mu \mathrm{m}$, they can be easily observed under a fluorescent or confocal microscope provided that the appropriate fluorescent probe was incorporated into the lipid phase during vesicle formation. GUVs can be formed from different lipid mixtures and they are stable in a wide range of physical conditions such as $\mathrm{pH}$, pressure or temperature. This mini-review presents information about the methods of GUV production and their usage. Particularly, the use of GUVs in studying lipid phase separation and the appearance and behavior of lipid domains (rafts) in membranes is discussed but also other examples of GUVs use in membrane research are given. The experience of the authors in setting up the GUV-forming equipment and production of GUVs is also presented.
\end{abstract}

Keywords: giant unilamellar vesicles, phase separation, lipid rafts, model membranes

\section{INTRODUCTION}

Model systems have been used since the very beginning of membrane research to get a better insight into the molecular origin of the observed membrane properties and structures. Most commonly used bilayer models, like bilayer/black lipid membranes (BLMs) or conventional uni- or multilamellar liposomes, were constructed in a way which excluded direct optical microscopic observation of the membranes. In the early nineteen eighties it was discovered that in appropriate conditions lipid swelling in aqueous media could produce unilamellar li- posomes of the average diameter reaching up to 100 $\mu \mathrm{m}$. Since the size of these liposomes was approximately two-three orders of magnitude greater than that of liposomes formed by other methods (sonification, extrusion etc.) they quickly got the name of giant unilamellar vesicles - GUVs. Formation of giant unilamellar liposomes by application of static (DC) electric field was reported for the first time by Angelova and Dimitrov (1986). The ability to observe the fluctuations of GUV surface by the optical microscope enabled to perform the experiments on the mechanical properties of the liposome membranes (Evans \& Kwok, 1982). GUVs were also used

${ }^{\square}$ Corresponding author: Andrzej B. Hendrich, Department of Biology and Medical Parasitology, Wrocław Medical University, Mikulicza-Radeckiego 9, 50-367 Wrocław, Poland; phone: (48) 71784 1511, fax: (48) 71784 0701; e-mail: hendrich@ biofiz.am.wroc.pl

Abbreviations: Chol, cholesterol; DAPC, diarachidoylphosphatidylcholine; DLPC, dilauroylphosphatidylcholine; DMPC, dimyristoylphosphatidylcholine; DMPE, dimyristoylphosphatidylethanolamine; DNPC, dinervonoylphosphocholine; DOPC, dioleoylphosphatidylcholine; DPPC, dipalmitoylphosphatidylcholine; DPPE, dipalmitoylphosphatidylethanolmine; DSPC, distearoylphosphatidylcholine; FCS, fluorescence correlation spectroscopy; GUV, giant unilamellar vesicle; $\mathrm{L}_{\mathrm{o}^{\prime}}$ liquid ordered phase; $\mathrm{L}_{\mathrm{d}}$ liquid disordered phase; PC, phosphatidylcholine; SM, sphingomyelin. 
in the studies aimed at elucidating the mechanisms of lipid swelling and liposome formation in aqueous media (Angelova \& Dimitrov, 1986; 1988). As found in subsequent experiments the range of lipid types that form GUVs in DC electric field was rather narrow, perhaps because the static field exerts only an "ordering" effect on lipid molecules. This limitation was overcome by a new experimental approach in which an alternating (AC) electric field was used in GUV formation (Angelova et al., 1992). The AC field, due to the permanent (usually sinusoidal) change of both direction and magnitude of the field intensity, apart from ordering effect also forces molecules to move, what might help lipids to spontaneously adopt bilayer packing and to form unilamellar structures. This new method enabled to form giant vesicles using a variety of lipid mixtures and to study different properties of their membranes. To give only a few examples of GUV investigations one can list the following studies. The mechanical properties of GUVs were determined by Needham et al. (1988a; 1988b) and by Angelova et al. (1992). GUV shape changes induced by different factors like osmotic pressure or $\mathrm{pH}$ changes were observed by Mathived et al. (1996). Two years later the study on the critical role of electrostatic repulsion during GUV formation was published by Akashi et al. (1998). In the majority of papers GUVs were used to study the properties and behavior of bilayers composed of different lipids. Apart from these studies there were investigations focused on the interactions of nonlipid components with liposomal membranes. Angelova et al. (1999) proposed GUVs as a model for direct microscopic observation of DNA interactions with lipid bilayers. Giant liposomes were also used in the study on the interactions of non-membrane proteins like DNase and RNase with lipid bilayers (Fischer et al., 2000). The investigation on the mechanism of electroporation performed by Riske and Dimova (2005) with the use of GUVs as a model membranes may serve as the last, but not least, example.
This mini-review is aimed at providing compact information about the use of giant unilamellar liposomes as a models enabling the direct microscopic observation of membrane domains, lipid rafts. We also present here our own experience in setting up the GUV-forming equipment and production of GUVs.

\section{GUV FORMATION IN AC ELECTRIC FIELD}

Electroformation of GUVs is the method allowing the reproducible production of giant liposomes and therefore is quite often used in laboratories performing studies on model bilayers. A short description of the set-up and methods used in GUVs experiments is based on the solutions recently introduced in our laboratory. This experimental set-up consists of the elements that are typically also used in other laboratories: the chamber with a temperature-controlling unit, AC electric generator (MCP type SG1639B) and a confocal microscope (Nikon, Eclipse TE2000-E with C1 confocal system). The chamber (see Fig 1A, B) and the temperature-controlling unit were designed and manufactured in the Department of Biophysics.

GUV preparation starts with covering the platinum electrodes with chloroform (or chloroform/ methanol) solution of the appropriate lipid mixture, also containing the fluorescent dye. Typically, the lipid concentration of $1.3 \mathrm{mM}$ is used and the solution contains $0.1 \mathrm{~mol} \%$ of the fluorescent probe. The electrodes are then dried under a stream of nitrogen, and the remaining chloroform is removed by placing the chamber in vacuum for at least $1 \mathrm{~h}$. After setting up the whole chamber lipids are hydrated by approx. $1 \mathrm{ml}$ of water. The temperature of the chamber is set to the value approx. $10^{\circ} \mathrm{C}$ higher than the temperature of the main phase transition of the highest-melting lipid used in the experiment. GUVs are produced using sinusoidal $10 \mathrm{~Hz}$ wave of the
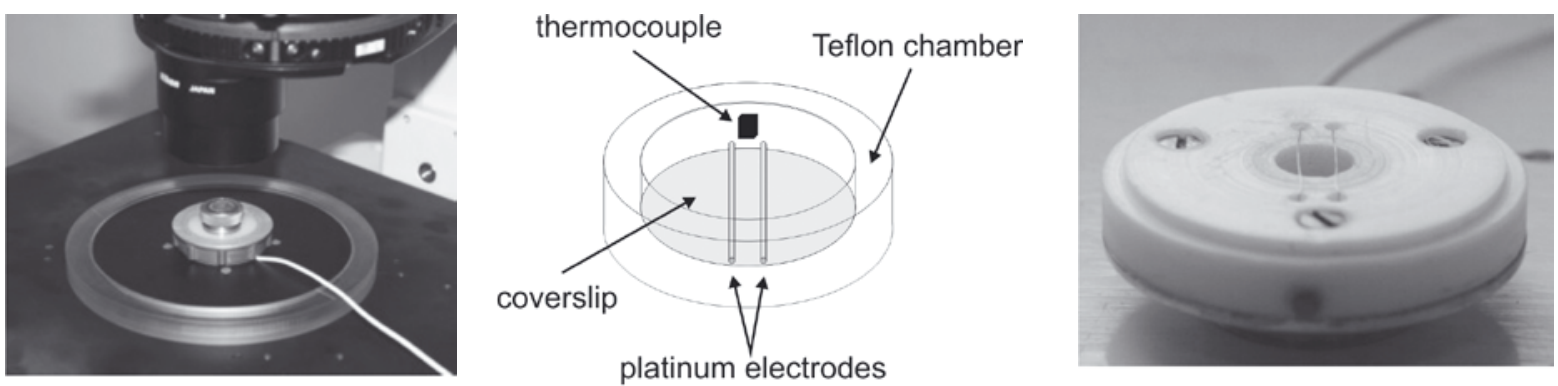

A
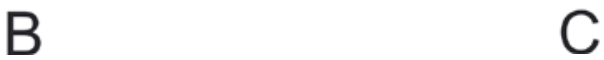

Figure 1. GUV formation chamber.

A. Chamber placed on the support of an inverted microscope. B. scheme of the chamber. C. Teflon part of chamber (note that the chamber is turned upside-down). 
amplitude of $3 \mathrm{~V}$ (peak-to-peak) applied for at least one hour to two parallel platinum-wire electrodes (diameter $0.1 \mathrm{~mm}$, placed at a distance of $2 \mathrm{~mm}$ ) located at the bottom of the Teflon cylindrical chamber presented in Fig. 1C. A thermocouple is placed in the wall of chamber, close to the electrodes. The temperature of the chamber is controlled with $0.2^{\circ} \mathrm{C}$ precision. In some other laboratories an indium tin oxide (ITO)-covered glass is used instead of the platinum electrodes. After the formation of GUVs the chamber temperature is lowered to the value at which the microscopic observations are carried out. GUV images are collected in either fluorescent or confocal mode of the microscope using the software supplied by the microscope manufacturer.

\section{OBSERVATION OF PHASE SEPARATION AND LIPID RAFTS}

Introduction of the $\mathrm{AC}$ electric field as a method of GUV formation has opened the way to produce giant vesicles made of lipid mixtures with natural propensity for phase separation and lipid raft formation. The domains emerging in GUV can be visualized using fluorescent probe(s) which bind preferentially to one of the phases present in the membrane. The fluorescent probe partitioning between $\mathrm{L}_{\mathrm{o}}$ and $\mathrm{L}_{\mathrm{d}}$ phases has been thoroughly studied and reviewed by Baumgart et al. (2007).

Phase separation could be easily observed in binary lipid mixtures when the mixture components significantly differ in main phase transition temperatures. When the system temperature is set to the value in the range between these transition temperatures one of the components is in the gel phase, and the second is in the liquid-crystalline state so that phase separation may occur. Appearance of domains in GUVs formed from binary lipid mixtures was observed for lipid compositions differing by acyl chain lengths, saturation or type of polar head-groups. Using AC electric field GUV formation method Bagatolli and Gratton (1999) have studied the occurrence of phase separation in giant vesicles formed from DPPC/DLPC and DPPE/DPPC lipid mixtures by means of two photon fluorescence microscopy (Bagatolli \& Gratton, 2000a). The domain coexistence was visualized using two types of fluorescent probes. Laurdan and Prodan belonged to the first type and they displayed different spectral properties in lipid gel and liquid-crystalline phases. $N$-Rh-DPPE (Lissamine rhodamine) represented the second type of fluorescent probe which partitioned preferentially into liquid disordered phase of the studied system (Baumgart et al., 2007). The shapes of domains observed in this work depended on the composition of lipids used to form GUVs and on the temperature. In DPPE/DPPC (7:3 mol/mol) mixtures in the temperature range corresponding to the phase coexistence $\left(41.3^{\circ} \mathrm{C}\right.$ to $\left.65^{\circ} \mathrm{C}\right)$ the dendritic domains, growing as temperature decreased, were recorded (see Fig. 2A). On the other hand, in DPPE/DPPC (3:7 $\mathrm{mol} / \mathrm{mol}$ ) mixtures the hexagonal (Fig. 2B) or sixcornered star (Fig. 2C) domains were observed. Hexagonal shapes of domains were also found by us in GUVs formed using DLPC/DPPC 1:1 lipid mixture (Fig. 3A). In case of this lipid mixture Bagatolli and Gratton (2000a) also recorded the appearance of line shapes (Fig. 2D), which presumably emerged due to the existence of linear defects in the bilayer structure. Dendritic domain shapes were also observed in DLPC/DAPC (1:1 $\mathrm{mol} / \mathrm{mol})$ and DMPE/DMPC (7:3 $\mathrm{mol} / \mathrm{mol}$ ) mixtures at the temperatures corresponding to the coexistence of different phases in those mixtures (Bagatolli \& Gratton, 2000b). The appearance of dendritic shapes was also demonstrated in single-component giant unilamellar vesicles formed from dinervonoylphosphocholine (DNPC) in the main phase-transition temperature region (Metso et al., 2005). These findings combined with the results of differential scanning calorimetric experiments (also presented in the above-mentioned paper) have confirmed that during the main phase transition of phospholipids the gel and liquid-crystalline domains co-exist in membranes.

The relation between appearance of different domain shapes and lipid compositions used in experiments is also summarized in Table 1.

Giant unilamellar DMPC/DSPC (1:1 mol/mol) vesicles were used in the study on the interactions of the polypeptide - gramicidin D with the lipid membranes (Fahsel et al., 2002). In the phase-coexistence temperature region the dendritic-like domains were observed in GUVs formed from DMPC/DSPC mixture. Like in the case of DPPE/DPPC mixtures described above the size of the domains increased as the temperature decreased. In the presence of 2 or $5 \mathrm{~mol} \%$ gramicidin the domains disappeared. Infrared spectroscopic measurements also performed in this work demonstrated that conformation of the polypeptide depended on the phase state and chain length of membrane lipids. The double helical gramicidin structure prevailed in DSPC membranes in the gel state while helical dimer structures were found in the fluid-crystalline DMPC domains. Due to the molecular sorting process in DSPC/DMPC mixtures in the phase-coexistence temperature region mostly the helical dimers were present in fluid DMPC domains.

Domain formation and vectorial vesicle budding induced by the asymmetrical sphingomyelinase-catalysed ceramide production in stearoyloleoylphosphatidylocholine (SOPC)/SM giant unilamellar liposomes was observed by Holopainen 
Table 1. Domain shapes and lipid compositions in which they appear

\begin{tabular}{lll}
\hline Domain shape & Lipid composition & Reference \\
\hline dendritic & DPPE/DPPC $(7: 3 \mathrm{~mol} / \mathrm{mol})$ & Bagatolli \& Gratton, 2000a \\
& DLPC/DPPC $(1: 1 \mathrm{~mol} / \mathrm{mol})$ & Bagatolli \& Gratton, 2000b \\
& DLPC/DAPC $(1: 1 \mathrm{~mol} / \mathrm{mol})$ & Bagatolli \& Gratton, 2000b \\
& DMPE/DMPC $(7: 3 \mathrm{~mol} / \mathrm{mol})$ & Bagatolli \& Gratton, 2000b \\
& DNPC (at phase transition temperature $)$ & Metso et al., 2005 \\
\hline hexagonal & DPPE/DPPC $(3: 7 \mathrm{~mol} / \mathrm{mol})$ & Bagatolli \& Gratton, 2000a \\
& DLPC/DPPC $(1: 1 \mathrm{~mol} / \mathrm{mol})$ & Bagatolli \& Gratton, 2000b \\
& DLPC/DSPC $(1: 1 \mathrm{~mol} / \mathrm{mol})$ & Bagatolli \& Gratton, 2000b \\
\hline star-like & DPPE/DPPC $(3: 7 \mathrm{~mol} / \mathrm{mol})$ & Bagatolli \& Gratton 2000a \\
& DMPC/DSPC $(1: 1 \mathrm{~mol} / \mathrm{mol})$ & Bagatolli \& Gratton, 2000b \\
& DLPC/DPPC $(1: 1 \mathrm{~mol} / \mathrm{mol})$ & Bagatolli \& Gratton, 2000b \\
\hline linear & DLPC/DPPC $(1: 1 \mathrm{~mol} / \mathrm{mol})$ & Bagatolli \& Gratton, 2000a, \\
& Bagatolli \& Gratton, 2000b \\
& DPPC/DOPC $(1: 1 \mathrm{~mol} / \mathrm{mol})$ & Scherfeld et al., 2003 \\
\hline circular & POPC/SM/Chol $(1: 1: 1 \mathrm{~mol} / \mathrm{mol} / \mathrm{mol})$ & Veatch \& Keller, 2003 \\
& DSPC/DOPC/Chol $(5: 5: 1 \mathrm{~mol} / \mathrm{mol} / \mathrm{mol})$ & Zhao et al., 2007 \\
& Kahya et al., 2006 \\
& Scherfeld et al., 2003 \\
& Kahya et al., 2005 \\
& DOPC/SM/Chol $(1: 1: 1 \mathrm{~mol} / \mathrm{mol} / \mathrm{mol})$ & Bacia et al., 2004 \\
& Bacia et al., 2005 \\
& Baumgart et al., 2007 \\
& Kahya et al., 2003
\end{tabular}

et al. (2000). According to the authors of this paper the appearance of ceramide microdomains in one of the bilayer leaflets generated spontaneous negative membrane curvature and led to the vesiculation of the bilayer. The vesiculation and ceramide transport seem to play a very important role in the cell signaling cascades involved in such processes as apoptosis, growth suppression and cell senescence.

Binary lipid mixtures make it possible to study the mixing properties of individual lipid species but bilayers formed from such mixtures do not resemble the membranes of eukaryotic cells where cholesterol (Chol) and sphingomyelin (SM) play an important role. Due to the presence of unsaturated phospholipids and large amounts of Chol in natural membranes instead of the gel or liquid-crystalline phases the liquid-ordered $\left(\mathrm{L}_{\mathrm{o}}\right)$ and liquid-disordered $\left(\mathrm{L}_{\mathrm{d}}\right)$ phases are observed. The $\mathrm{L}_{\mathrm{o}}$ domains rich in Chol and SM are commonly called the lipid rafts. The ability of straightforward visualization of structures resembling the lipid rafts prompted scientists to perform GUV experiments using ternary lipid mixtures containing different phospholipids, SM and Chol (so called "raft mixtures"). In one of the first studies the presence of circular domains was demonstrated in free standing membranes of GUVs formed of equimolar DOPC/SM/Chol mixtures as well as in supported planar bilayers formed of POPC/SM/Chol mixtures (2:1:1) (Dietrich et al., 2001). This study also confirmed directly that raft domains were co-localized in both inner and outer vesicle leaflets and inter-bilayer domain coupling occurred. An almost perfectly circular shape of the observed domains (Fig. 2E) also supported the possibility of the co-existence of two fluid (liquid-ordered and liquid disordered) phases in the raft-like lipid mixtures.

Since Chol is an important structural component of natural membranes some research groups studied the role it played in the process of raft formation in GUVs. In giant unilamellar vesicles formed from lipid mixtures containing one saturated and one unsaturated lipid the formation of circular domains was observed when Chol contribution was changed from $5 \mathrm{~mol} \%$ up to $50 \mathrm{~mol} \%$ of sample (Veatch \& Keller, 2002). Simultaneously in EYPC/SM/Chol mixtures the presence of circular domains was recorded in narrower Chol content range (20 $\mathrm{mol} \%$ to $40 \mathrm{~mol} \%$ ). In the next publication the same authors studied the wide range of ternary lipid mixtures and the phase diagram was constructed for one particular mixture: DPPC/DOPC/Chol (Veatch \& Keller, 2003). A comprehensive study on the role of GUV composition on the raft formation was published by the research group of Kahya and coworkers $(2003 ; 2004)$. They used the giant liposomes made of DOPC/SM/Chol mixed in different proportions to follow the role of individual components in lipid raft formation. It was found that Chol played a key role in the promotion of lipid segregation and phase separation (lipid rafts) were observed exclusively in a certain range of Chol concentrations (10-50 mol\%). As for the previously described phospholipid mixtures the domains observed in presence of Chol were almost perfectly circular. In the 

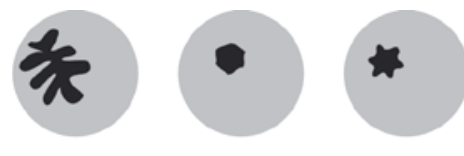

A

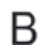

C
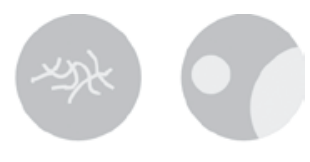

D

E

Figure 2. The basic types of domains observed for different lipid mixtures used to produce GUVs.

A. Dendritic (DPPE/DPPC). B and C. Hexagonal or starlike (DLPC/DPPC). D. Linear (DLPC/DPPC). E. Circular (POPC/SM/Chol).

other study carried out by the same research group (Scherfeld et al., 2003) the propensity of ternary lipid mixtures containing either SM or saturated phosphatidylcholines (PCs) to form domains was compared. Comparing the ternary mixtures containing (apart from Chol and unsaturated PC) either SM or saturated PCs it was shown that in systems containing saturated PCs more Chol was needed to observe phase separation. Additionally Scherfeld et al. (2003) using fluorescence correlation spectroscopy (FCS) confirmed that Chol-PC interactions were weaker than Chol-SM ones.

SM was "replaced" by saturated phospholipids also in another study in which the ternary phase diagram for DSPC/DOPC/Chol mixtures was constructed (Zhao et al., 2007). The appearance of circular domains was observed in this case for Chol concentrations ranging from $15 \mathrm{~mol} \%$ up to $40 \mathrm{~mol} \%$. Similarity of the results obtained for lipid mixtures containing apart from Chol also either SM or another high melting temperature phospholipid (DSPC) suggested that not only specific Chol-SM interactions played an important role in lipid raft formation.

The role of Chol in domain formation in GUVs produced from Chol-containing ternary lipid mixtures was also confirmed by Chol-depletion experiments (Bacia et al., 2004). It was shown by confocal microscopy and fluorescence correlation spectroscopy that $\mathrm{L}_{\mathrm{o}}$ domains, which were present in Chol-rich giant vesicles, disappeared when the sterol was removed from membranes by methyl- $\beta$ cyclodextrin treatment. In the next study the same research group proved that apart from $\mathrm{Chol}$ also the presence of other sterols determined the phase separation phenomena in GUVs (Bacia et al., 2005) and the type of observed effects depended on the chemical structure of individual sterols. Out of five sterols studied ketocholesterol showed no domain-inducing properties, Chol and lophenol induced domains and caused outward vesicle budding while lanosterol and cholesteryl sulphonate apart from domains induced inward budding. The different budding directions seemed to be dependent on the ability of individual sterols to flip-flop between the bilayer leaflets. Sterols characterized by fast flip-flop rate caused out-
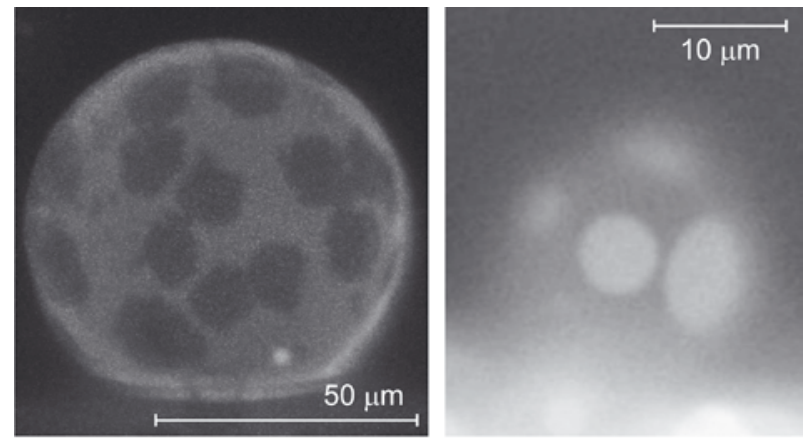

A

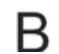

Figure 3. Shapes of domains observed in our laboratory. A. Hexagonal (DLPC/DPPC 1:1 and 1,1'-dioctadecyl3,3,3', $3^{\prime}$-tetramethylindocarbocyanine perchlorate, $\operatorname{DiIC}_{18}(3)$, as fluorescent probe), image obtained by confocal microscopy. B. Circular (DOPC/SM/Chol 1:1:1 and 1,1'-didodecyl-3,3,3', 3'-tetramethylindocarbocyanine perchlorate, $\mathrm{DiIC}_{12}(3)$, as fluorescent probe), image obtained by fluorescence microscopy. Confocal and fluorescence images were obtained using Nikon Eclipse TE2000-E microscope with C1 confocal system.

ward budding and those which flip-flop slowly presumably induced inward budding. GUVs were also used in the experiments in which the effects exerted by motions of other membrane components on the bilayer curvature were studied. The impact of shortand long-chain ceramide transbilayer movements on the membrane shape was studied by Lopez-Montero et al. (2005). Surprisingly, the domain (raft?) formation and/or enlargement could also be induced by light in certain ternary lipid mixtures. This phenomenon was first reported by Ayuyan and Cohen (2006) who found that in GUVs made of DOPC/SM/ Chol mixtures the light-induced lipid peroxidation triggers the process of domain enlargement.

In certain cases the presence of Chol in the studied systems induced the formation of tubular networks between the vesicles (Nomura et al., 2005). Neuron-like tubes connecting neighbouring giant unilamellar vesicles formed from DOPC and EYPC were observed in systems containing 5-30 $\mathrm{mol} \%$ of Chol. One should emphasize, however, that in those experiments giant liposomes were prepared by simple swelling, not by the electroformation method.

Even though this is outside the scope of this mini-review it is worth mentioning that giant unilamellar vesicles also serve as a model membranes in the studies on interactions of proteins (Fischer et al., 2000; Fahsel et al., 2002; Staneva et al., 2004), oligonucleotides (Gordon et al., 2005), detergents (Tamba et al., 2004; Staneva et al., 2005), ethanol (Tierney et al., 2005) or local anesthetics (Lee et al., 2007) with lipids. Due to the proper reconstitution technique proteins incorporated into GUV membranes were 
reported to retain $100 \%$ of their activity (Doeven et al., 2005).

Since their existence in cell membranes was first proposed by Simons and Ikonen (1997) lipid rafts have attracted the attention of many laboratories. It seems that they are involved in a variety of cellular processes like signaling, membrane transport, trafficking and many others. Despite this popularity of lipid rafts, the knowledge about the molecular basis of their existence is not complete and some questions about their size, composition and life span remain open (Kenworthy, 2005). As we tried to show above the giant unilamellar vesicles may serve as an ideal tool in the studies devoted to lipid rafts. GUVs can be formed from well known lipid mixtures and their composition can mimic the natural membranes. The size of GUVs enables direct optical microscopic observations, additionally the usage of advanced experimental techniques like confocal microscopy or fluorescence correlation spectroscopy allows to study both static structural and dynamical properties of these systems. Therefore we believe that GUVs will remain one of the most important model systems used in membrane studies.

\section{Acknowledgement}

This work was supported by a grant 2 P05A 09020 from the Polish Ministry of Science and Higher Education.

\section{REFERENCES}

Akashi K, Miyata H, Itoh H, Kinosita K Jr (1996) Preparation of giant liposomes in physiological conditions and their characterization under an optical microscope. Biophys J 71: 3242-3250.

Angelova MI, Dimitrov DS (1986) Liposome electroformation. Faraday Discuss Chem Soc 81: 303-311.

Angelova MI, Dimitrov DS (1988) A mechanism of liposome electroformation. Prog Colloid Polymer Sci 76: 59-67.

Angelova MI, Tsoneva I (1999) Interactions of DNA with giant liposomes. Chem Phys Lipids 101: 123-137.

Angelova MI, Soleau S, Meleard Ph, Faucon JF, Bothorel F (1992) Preparation of giant vesicles by external AC electric fields. Kinetics and applications. Progr Colloid Polymer Sci 89: 127-131.

Ayuyan AG, Cohen FS (2006) Lipid peroxides promote large rafts: effects of excitation of probes in fluorescence microscopy and electrochemical reactions during vesicle formation. Biophys J 91: 2172-2183.

Bacia K, Scherfeld D, Kahya N, Schwille P (2004) Fluorescence correlation spectroscopy relates rafts in model and native membranes. Biophys J 87: 1034-1043.

Bacia K, Schwille P, Kurzchalia T (2005) Sterol structure determines the separation of phases and the curvature of the liquid-ordered phase in model membranes. Proc Natl Acad Sci USA 102: 3272-3277.

Bagatolli LA, Gratton E (1999) Two-photon fluorescence microscopy observation of shape changes at the phase transition in phospholipid giant unilamellar vesicles. Biophys J 77: 2090-2101.

Bagatolli LA, Gratton E (2000a) Two photon fluorescence microscopy of coexisting lipid domains in giant unilamellar vesicles of binary phospholipid mixtures. Biophys J 78: 290-305.

Bagatolli LA, Gratton E (2000b) A correlation between lipid domain shape and binary phospholipid mixture composition in free standing bilayers: A two-photon fluorescence microscopy study. Biophys J 79: 434-447.

Baumgart T, Hunt G, Farkas ER, Webb WW, Feigenson GW (2007) Fluorescence probe partitioning between Lo/Ld phases in lipid membranes. Biochim Biophys Acta 1768: 2182-2194.

Dietrich C, Bagatolli LA, Volovyk ZN, Thompson NL, Levi M, Jacobson K, Gratton E (2001) Lipid rafts reconstituted in model membranes. Biophys J 80: 1417-1428.

Doeven M, Folgering JH, Krasnikov V, Geertsma ER, van den Bogaart G, Poolman B (2005) Distribution, lateral mobility and function of membrane proteins incorporated into giant unilamellar vesicles. Biophys J 88: 11341142.

Evans E, Kwok R (1982) Mechanical calorimetry of large dimyristoylphosphatidylcholine vesicles in the phase transition region. Biochemistry 21: 4874-4879.

Fahsel S, Pospiech EM, Zein M, Hazlet TL, Gratton E, Winter R (2002) Modulation of concentration fluctuations in phase-separated lipid membranes by polypeptide insertion. Biophys J 83: 334-344.

Fischer A, Oberholzer T, Luisi PL (2000) Giant vesicles as models to study the interactions between membranes and proteins. Biochim Biophys Acta 1467: 177-188.

Gordon S, Berezhna S, Scherfeld D, Kahya N, Schwille P (2005) Characterization of interaction between cationic lipid-oligonucleotide complexes and cellular membrane lipids using confocal imaging and fluorescence correlation spectroscopy. Biophys J 88: 305-316.

Holopainen J, Angelova MI, Kinnunen PK (2000) Vectorial budding of vesicles by asymmetrical enzymatic formation of ceramide in giant liposomes. Biophys J 78: 830-838.

Kahya N, Scherfeld D, Bacia K, Poolman B, Schwille P (2003) Probing lipid mobility of raft-exhibiting model membranes by fluorescence correlation spectroscopy. J Biol Chem 278: 28109-28115.

Kahya N, Scherfeld D, Bacia K, Schwille P (2004) Lipid domain formation and dynamics in giant unilamellar vesicles explored by fluorescence correlation spectroscopy. J Struct Biol 147: 77-89.

Kenworthy AK (2005) Fleeting glimpses of lipid rafts: how biophysics is being used to track them. J Investig Med 53: 312-317.

Lee YH, Park NS, Kwon JD, Park JS, Shin GB, Lee CS, Jung TS, Choi NJ, Yoon JH, Ok JS, Yoon UC, Bae MK, Jang $\mathrm{HO}$, Yun I (2007) Amphiphilic effects of dibucaine. $\mathrm{HCl}$ on rotational mobility of $\mathrm{n}$-(9-anthroyloxy)stearic acid in neuronal and model membranes. Chem Phys Lipids 146: $33-42$.

Lopez-Montero I, Rodriguez N, Cribier S, Pohl A, Velez M, Devaux PF (2005) Rapid transbilayer movement of ceramides in phospholipid vesicles and in human erythrocytes. J Biol Chem 280: 25811-25819.

Mathivet L, Cribier S, Devaux PF (1996) Shape change and physical properties of giant phospholipid vesicles prepared in the presence of an AC electric field. Biophys J 70: $1112-1121$.

Metso AJ, Zhao H, Tuunainen I, Kinnunen PK (2005) Observation of the main phase transition of dinervonoyl- 
phosphocholine giant liposomes by fluorescence microscopy. Biochim Biophys Acta 1713: 83-91.

Needham D, Evans E (1988a) Structure and mechanical properties of giant lipid (DMPC) vesicle bilayers from 20 degrees $C$ below to 10 degrees $C$ above the liquid crystal-crystalline phase transition at 24 degrees C. Biochemistry 27: 8261-8269.

Needham D, McIntosh TJ, Evans E (1988b) Thermomechanical and transition properties of dimyristoylphosphatidylcholine/cholesterol bilayers. Biochemistry 27: 4668-4673.

Nomura SM, Mizutani Y, Kurita K, Watanabe A, Akiyoshi $\mathrm{K}$ (2005) Changes in the morphology of cell-size liposomes in the presence of cholesterol: formation of neuron-like tubes and liposome networks. Biochim Biophys Acta 1669: 164-169.

Riske KA, Dimova R (2005) Electro-deformation and poration of giant vesicles viewed with high temporal resolution. Biophys J 88: 1143-1155.

Scherfeld D, Kahya N, Schwille P (2003) Lipid dynamics and domain formation in model membranes composed of ternary mixtures of unsaturated and saturated phosphatidylcholines and cholesterol. Biophys J 85: 37583768.

Simons K, Ikonen E (1997) Functional rafts in cell membranes. Nature 387: 569-572.

Staneva G, Angelova MI, Koumanov K (2004) Phospholipase A2 promotes raft budding and fission from giant liposomes. Chem Phys Lipids 129: 53-62.
Staneva G, Seigneuret M, Koumanov K, Trugnan G, Angelova MI (2005) Detergents induce raft-like domains budding and fission from giant unilamellar heterogeneous vesicles. A direct microscopy observation. Chem Phys Lipids 136: 55-66.

Tamba Y, Tanaka T, Yahagi T, Yamashita Y, Yamazaki M (2004) Stability of giant unilamellar vesicles and large unilamellar vesicles of liquid-ordered phase membranes in the presence of Triton X-100. Biochim Biophys Acta 1667: 1-6.

Tierney KJ, Block DE, Longo ML (2005) Elasticity and phase behavior of DPPC membrane modulated by cholesterol, ergosterol, and ethanol. Biophys J 89: 24812493.

Veatch SL, Keller SL (2002) Organization in lipid membranes containing cholesterol. Phys Rev Lett 89: 2681011-268101-4.

Veatch SL, Keller SL (2003) Separation of liquid phases in giant vesicles of ternary mixtures of phospholipids and cholesterol. Biophys J 85: 3074-3083.

Zhao J, Wu J, Heberle FA, Mills TT, Klawitter P, Huang G, Costanza G, Feigenson GW (2007) Phase studies of model biomembranes: complex behavior of DSPC/ DOPC/cholesterol. Biochim Biophys Acta 1768: 27642776. 